\title{
L'enseignement de l'EPS à des élèves avec une déficience intellectuelle : aspects organisationnels et pédagogiques
}

Jean-Pierre GAREL ${ }^{1}$

Discipline d'enseignement, l'EPS a sa place dans tous les lieux de scolarisation, dans les établissements médico-éducatifs et sanitaires, sous tutelle du ministère en charge de la santé, et bien sûr dans les établissements relevant de l'Éducation nationale, qui accueillent un nombre croissant d'enfants et d'adolescents en situation de handicap, individuellement au sein de classes ordinaires ou dans le cadre des dispositifs d'intégration collective, les Clis pour le premier degré et les Ulis² pour le second ${ }^{3}$.

Les déficiences intellectuelles caractérisent un grand nombre de jeunes handicapés scolarisés à l'Éducation nationale ${ }^{4}$, et pourtant, en termes de formation de leurs enseignants d'EPS, ils sont l'objet d'une moindre attention que les élèves présentant une déficience motrice ou sensorielle 5 .

\footnotetext{
${ }^{1}$ Chercheur associé, EA 4110, ER3S, PRES Lille Nord de France.

${ }^{2}$ La circulaire $n^{\circ} 2010-088$ du 18-6-2010, parue au Bulletin officiel n ${ }^{\circ} 28$ du 15 juillet 2010, indique qu'à compter du 1er septembre 2010 les dispositifs collectifs implantés en collège et en lycée, jusque-là intitulés Unités pédagogiques d'intégration (UPI), sont dénommés Unités localisées pour l'inclusion scolaire (Ulis). ${ }^{3}$ À la rentrée scolaire 2009, 187500 élèves porteurs de maladies invalidantes ou de handicaps étaient accueillis dans les écoles et établissements scolaires ordinaires (Données du M.E.N. : Le système éducatif. Repères et références statistiques - édition 2010) contre 103000 en 1999 (selon la DREES, Études et résultats, № 216, janvier 2003).

${ }^{4}$ Les élèves souffrant de « troubles intellectuels et cognitifs », qui comprennent, selon la classification retenue dans les Données du M.E.N. citées, les troubles envahissants du développement, dont l'autisme, constituent 48 $\%$ des effectifs dans le premier degré et $36 \%$ dans le second degré.

${ }^{5}$ Voir les contenus des stages académiques de formation continue des enseignants et ceux des sites académiques dédiés à l'EPS sur le web.
} 
Il faut dire que leurs troubles ne s'accompagnent pas d'altérations corporelles aussi manifestes et ne laissent donc pas présager des problèmes importants en EPS. En fait, cette discipline peut être source de difficultés pour des jeunes porteurs de ces troubles et mettre les enseignants dans l'embarras, alors qu'elle peut aussi constituer pour ces élèves une plage de réussite intéressante, tant pour les apprentissages qu'elle permet que pour la vie commune et l'intégration qu'elle favorise.

Pour que ces jeunes puissent retirer de cet enseignement les bénéfices espérés, des éléments d'information, de réflexion et de préconisation sont, pour le moins, nécessaires à leurs professeurs. C'est à en livrer quelques-uns que nous nous attacherons ici, en nous appuyant sur des connaissances issues de la recherche, d'observations et d'expériences. Trois étapes de l'intervention seront abordées : l'élaboration du projet d'EPS, la préparation des séances et l'accompagnement de l'élève dans son activité.

\section{L'ELABORATION DU PROJET}

Le projet d'EPS destiné à un élève avec une déficience intellectuelle, accueilli en établissement scolaire ordinaire, s'inscrit dans le cadre du projet personnalisé de scolarisation (PPS) ${ }^{6}$. À partir des informations qu'il contient, d'une évaluation «diagnostique » de l'élève à laquelle il a pu procéder, et en se fondant si besoin sur d'autres éléments de connaissance du jeune, éventuellement obtenus du jeune lui-même, de ses proches et des professionnels qui interviennent auprès de lui, le professeur d'EPS élabore un projet spécifique pour cet élève et cette discipline, en cohérence avec les partenaires qui peuvent être impliqués dans le projet. À côté des moyens, d'ordre matériel ou humain, qui sont éventuellement à prévoir, par exemple en termes de présence et de rôle d'un AVS — auxiliaire de vie scolaire — ou de l'enseignant de l'Ulis lors de séances d'EPS, le projet s'attache notamment à définir des objectifs, la programmation des activités et les modalités de groupement des élèves.

\subsection{La détermination d'objectifs}

Dès cette étape, si la connaissance des besoins de l'élève considéré est suffisante, fondée sur l'identification de ses capacités comme de ses difficultés, des objectifs adaptés à ses caractéristiques personnelles peuvent être formulés, concernant des ressources à développer, des connaissances et des compétences à acquérir (Garel, 2009). Au-delà d'une prise en compte de la singularité individuelle, les finalités et les objectifs généraux de l'EPS, tels qu'ils sont définis dans les textes officiels de l'Éducation nationale, s'appliquent aux élèves en situation de handicap comme aux autres.

La finalité d'intégration — ou d'inclusion, si l'on souhaite reprendre ce terme actuel souvent prégnante dans l'expression des finalités de l'intervention auprès de cette population particulière, ne doit pas occulter ce qui est propre à l'EPS : l'acquisition de pouvoirs corporels.

\footnotetext{
${ }^{6}$ Voir la circulaire n ${ }^{\circ}$ 2006-126 du 17 août 2006, relative à la mise en œuvre et au suivi du projet personnalisé de scolarisation.
} 
Certes, faire en sorte que ces jeunes ne demeurent pas à côté des autres sans interagir avec eux dans des situations suffisamment valorisantes et socialisantes est bien un objectif à retenir, mais si le vivre ensemble est important, il est à travailler à partir des situations motrices et des apprentissages auxquels elles peuvent donner lieu, pas à la place. L'adaptation à ces élèves ne doit pas se traduire sur ce plan par une moindre exigence à leur égard.

\subsection{La programmation des activités}

Le souci de favoriser l'intégration des personnes en situation de handicap conduit à programmer, dans la mesure du possible, des activités semblables à celles des valides. À l'opposé d'activités à visée rééducative, relevant d'une conception médicale du handicap, il s'agit de s'orienter vers une conception sociale et de privilégier des activités physiques, sportives et artistiques (APSA) qui sont des pratiques sociales valorisées et valorisantes, transmises, ou susceptibles de l'être, d'une génération à l'autre, et qui permettent à ceux qui s'y adonnent de partager une culture commune et de développer un sentiment d'appartenance à une communauté élargie. Cela dit, certaines activités peuvent s'avérer plus pertinentes que d'autres (Brunet \& Mautuit, 2003). Si, par exemple, un objectif retenu dans le projet de l'élève porte sur l'amélioration du contrôle de l'équilibre dynamique et statique, l'escalade, la gymnastique et les arts du cirque offrent des possibilités de travail intéressantes.

L'intention de favoriser les relations entre élèves peut trouver à s'opérationnaliser dans de multiples activités, bien au-delà des sports collectifs. On peut même dire que, dans le cadre d'une pratique mixte, ce type de sports est parfois source de problèmes pour des jeunes avec un retard mental. En effet, l'insuffisance de leurs ressources cognitives peut les mettre en difficulté lorsque la réussite dépend pour beaucoup des informations et des décisions prises dans un contexte où il faut agir rapidement. C'est pourquoi des enseignants d'EPS du collège Pierre Mendès France, à Riom, ont décidé de ne pas proposer, en situation d'intégration, un sport aussi exigeant, sur le plan cognitif, que le basket-ball. En revanche, un jeu collectif comme la thèque, qui sollicite moins les ressources qui leur font défaut, est retenu (Garel, 2001)'.

Par ailleurs, la présence de jeunes en situation de handicap au sein d'une équipe risque d'être mal acceptée lorsque l'importance de la victoire est (trop) fortement ressentie par les protagonistes et que des partenaires de faible niveau exposent à une défaite. Plus généralement, un affrontement entre individus ou équipes qui est caractérisé par un fort déséquilibre des forces en présence est dommageable pour les plus faibles, sauf à mettre en œuvre des adaptations profitables à tous et suffisamment bien acceptées.

À l'inverse, des activités physiques artistiques, qui ne mettent pas l'opposition au premier plan, offrent une diversité de rôles tels que tout élève a des chances d'en assumer un à sa mesure et de s'inscrire dans un projet collectif riche de liens entre les membres du groupe.

\footnotetext{
${ }^{7}$ Le choix d'écarter le basket-ball, décidé dans un contexte local, ne signifie pas que cette activité doive être $a$ priori supprimée pour des élèves avec une déficience intellectuelle. L'hétérogénéité de la population considérée est trop vaste pour que l'on puisse se dispenser de fonder le choix des activités sur une observation attentive des possibilités de ces élèves.
} 


\subsection{L'affectation à un groupe d'élèves}

L'élève peut participer aux cours d'EPS selon différentes modalités de groupement :

- avec sa classe de référence ;

- avec une autre classe, jugée mieux adaptée si, par exemple, son effectif est moins élevé, si elle pose moins de problèmes à l'enseignant, si elle comporte un autre jeune dont l'élève se sent proche, en particulier parmi ceux qui relèvent d'une Ulis, ou si l'activité qui y est programmée lui convient mieux ;

- au sein d'un groupe composé exclusivement d'élèves en situation de handicap mental, pour une pratique entre soi. C'est une modalité que l'on rencontre notamment lorsqu'une Ulis est implantée dans l'établissement.

Une autre distinction peut être identifiée, entre intégration individuelle et intégration collective ; un avantage de cette seconde modalité, caractérisée par la présence dans un même groupe de plusieurs élèves en situation de handicap, étant notamment qu'elle peut s'avérer plus rassurante pour certains des élèves ainsi accueillis (Joly, 2006).

Le collège Pierre Mendès France illustre une organisation faisant coexister différentes modalités : chacun des adolescents de l'Ulis a la possibilité d'un temps d'activité entre soi et d'un temps de pratique mixte que l'on rencontre sous deux formes :

- en intégration individuelle : un nombre restreint de ces adolescents participent régulièrement aux cours d'EPS avec les autres élèves d'une classe ordinaire ;

- en intégration collective : tous les élèves de l'Ulis, sauf ceux pour qui le projet pédagogique et éducatif prévoit une intégration individuelle, sont intégrés dans une même classe pour certains cycles d'activités ou pour une activité ponctuelle.

Le choix d'intégrer tel élève avec telle classe se décide en prenant notamment en compte le profil de l'élève, les caractéristiques de la classe d'accueil et la programmation des APSA.

\section{LA PREPARATION DES SEANCES D'EPS : DIFFERENCIER AUTANT QUE DE BESOIN}

La présence d'un enfant ou d'un adolescent en situation de handicap au sein d'un groupe d'élèves ordinaires appelle des différenciations lui permettant de progresser et d'interagir avec le maximum de chances de réussite, c'est-à-dire qui ne l'enferment pas dans un traitement distinctif marquant un manque d'ambition pédagogique et éducative.

Les différenciations peuvent concerner les objectifs et les contenus d'enseignement. Elles se traduisent aussi par l'adaptation des tâches. Après avoir considéré leur difficulté objective en regard des caractéristiques propres de l'élève, on peut jouer sur leurs variables - environnement physique et humain, règles, etc.

\subsection{Des objectifs aux contenus d'enseignement}

Pour une séance donnée, les objectifs généraux formulés dans le projet personnalisé demandent à être précisés, opérationnalisés, pour être adaptés à une APSA particulière et aux obstacles 
qu'y rencontre l'élève pour progresser. Le choix de ces objectifs-obstacles, ainsi qualifiés par Philippe Meirieu puisqu'y satisfaire " permet au sujet de franchir un palier décisif de progression » $(1987,184)$, aboutit logiquement à définir des contenus d'enseignement adaptés à l'élève considéré, c'est-à-dire ce qu'il va être conduit à apprendre. Au basket, par exemple, que privilégier : le dribble de progression ou de dépassement, le tir en course, les choix du porteur du ballon, le démarquage ? Si l'on retient le tir en course, on constatera peut-être que la coordination de la course et du dribble, ou l'enchaînement de la course en dribblant et du tir, est d'un niveau de complexité trop élevé. Et l'on devra alors décomposer l'ensemble en actes plus simples.

À cet objectif d'apprentissage centré sur ce qui est prioritaire, on pourra ajouter ce que l'élève doit faire pour réussir, ce qui peut s'exprimer par une formulation du type : pour arriver à..., il faut... (Garel, 2009).

Ce travail didactique ne doit pas occulter la nécessaire compréhension de ce qui fait sens aux yeux d'un élève. La recherche de performance est peut-être pour lui, d'entrée de jeu, peu motivante. Le plaisir sensorimoteur et les émotions vécues peuvent, pendant quelque temps, prendre le pas sur toute autre considération. Il est donc important d'identifier le type d'entrée dans une activité qui est susceptible de le mobiliser. Dans les arts du cirque, par exemple, les acrobaties, les manipulations d'objets, les activités d'expression et de communication, le travail individuel ou avec des partenaires, constituent une diversité de voies et de contextes de travail dans lesquels un élève est susceptible de trouver des motifs pour s'engager.

\subsection{L'environnement physique}

Selon des recherches scientifiques popularisées notamment par Jean-Pierre Famose, un enseignement s'appuyant sur des démonstrations et des explications pour présenter aux élèves ce qui est à effectuer est moins efficace qu'une stratégie consistant à les laisser rechercher la solution de façon autonome. Dans le domaine des activités physiques adaptées aux personnes avec un retard mental, les méthodes pédagogiques prônées vont souvent dans ce sens : elles « doivent valoriser les apprentissages qualifiés d'auto-adaptatifs, c'est-à-dire réalisés à partir de milieux riches et aménagés. Dans ces situations d'apprentissage, le sujet s'auto-organise en fonction des sollicitations du milieu » (Brunet, Bui-Xuân \& Bluteau, 2001, 140).

Ces méthodes accordent une place de choix à un aménagement matériel de l'environnement, susceptible d'induire ce qui est attendu. Le fait que l'élève puisse conduire son activité hors du regard de l'enseignant et qu'il prenne par lui-même connaissance du résultat atteint, à partir d'indices concrets issus de l'environnement, concourt à son autonomie.

Chez les personnes en situation de handicap mental, une "perception chaotique de l'environnement » (Magerotte, 1984) peut être améliorée en le structurant par des repères qui composent des formes simples et qui sont susceptibles de limiter et d'orienter l'espace d'action : plots, nattes ou tapis fournissant des informations visuelles et tactilo-kinesthésiques, manuelles ou plantaires, etc. De multiples éléments peuvent ainsi être disposés pour provoquer des sollicitations multisensorielles. Comme pour tous les élèves, l'aménagement de l'environnement stable est conçu de manière à l'adapter aux possibilités individuelles : 
réduction de la surface du terrain au badminton, diminution de la hauteur et de la distance séparant les obstacles d'un parcours...

Quant à la difficulté de l'environnement incertain, caractérisé par la mobilité des éléments en jeu - objets ou individus - , elle peut être modulée en jouant sur leur nombre - de joueurs, par exemple —, leur clarté — balle de couleur vive...-, leur volume - grosse balle plus visible et moins rapide qu'une petite...-, leur composition — balle en mousse peu rapide leurs caractéristiques spatio-temporelles - la trajectoire d'une balle est plus facile à apprécier quand elle roule que lorsqu'elle rebondit...

Enfin, les objets manipulés constituent une variable qui joue sur la difficulté des tâches. Ainsi, au tennis de table, une grande raquette accroît les chances de frapper la balle.

\subsection{L'environnement humain}

Si la question de l'affectation d'un élève à un groupe-classe ordinaire ou à un groupe spécifique a déjà été envisagée lors de l'élaboration du projet individuel, elle se pose à nouveau quand il s'agit de répartir les élèves en groupes restreints, pour une séance et une activité données.

Dans une perspective d'intégration, lorsque plusieurs élèves avec une déficience intellectuelle sont accueillis dans une classe ordinaire, les groupements peuvent être choisis de façon à éviter que les jeunes en situation de handicap soient exclusivement entre eux. Ainsi, au collège La Bucaille, lors des activités collectives, les équipes sont hétérogènes et homogènes entre elles, et quand il est demandé aux élèves de se regrouper par deux ou trois, les adolescents de l'Ulis ne doivent pas se retrouver ensemble (Joly, op. cit.).

Pourtant, il est parfois pertinent que ces jeunes soient réunis, non sur la base de leur déficience, mais en fonction de besoins qui se révèlent au fil des séances d'EPS. Des ateliers peuvent ainsi être organisés, qui ont pour objet de développer une capacité physique, une compétence technique, ou de remédier à des difficultés. S'y retrouvent les élèves partageant de mêmes besoins, en situation de handicap ou non.

Par ailleurs, les regroupements doivent être considérés à l'occasion d'activités où il est important que l'élève handicapé soit avec un (des) partenaire (s) présentant à la fois un niveau sportif suffisant et une attitude coopérative. Dans les sports de raquettes, par exemple, il est intéressant que le partenaire soit un bon relanceur, capable de limiter la rupture de l'échange.

L'environnement humain est également à envisager du point de vue des aides que les élèves ordinaires peuvent apporter aux jeunes en situation de handicap. Le collège Pierre Mendès France en fournit une illustration : en gymnastique, chaque groupe de travail comprend deux élèves de l'Ulis, dont les autres ont la responsabilité, et, lors du jeu de thèque, la difficulté à se repérer dans l'espace est palliée par l'appariement de certains élèves de l'Ulis avec des camarades de la classe de cinquième.

Dans un autre collège, le tutorat est organisé de façon systématique : l'élève handicapé désigne dans la classe d'accueil un camarade référent, volontaire, auquel il peut éventuellement recourir, notamment pour se faire expliquer ce qu'il n'a pas compris.

Au collège Maryse Bastié, à Décines, le professeur d'EPS ayant constaté, en lutte, la difficulté 
d'élèves de l'Ulis à réinvestir des techniques bien maitrisées, confie à un élève ordinaire le soin d'accompagner un camarade handicapé dans les situations de combat, pour l'encourager, lui rappeler le but à atteindre et donner des conseils - «écarte ta jambe, appuie avec ton ventre », etc.

\subsection{Les règles de l'activité}

Dans un souci d'équité, les élèves en situation de handicap peuvent bénéficier de règles spécifiques, en prenant garde que cette faveur ne soit pas une entrave à des progrès qui sont à leur portée. L'inégalité des droits propres à une activité vise l'égalité des chances. Au basketball, par exemple, on peut accorder à certains joueurs un point si leur tir touche le dessus de l'anneau du panier, interdire à leurs adversaires de défendre trop près d'eux sur l'ensemble du terrain ou lors d'un tir au panier. Ces règles de jeu particulières facilitent l'accessibilité de ce sport et permet un meilleur équilibre des forces en présence.

D'autres règles peuvent avoir des conséquences notables sur l'organisation des équipes et les relations entre élèves : un professeur du collège J. Macé, à Mainvilliers, attribue un bonus de quinze points à l'équipe de basket-ball dont tous les joueurs ont marqué au moins un point. De ce fait, les adolescents handicapés osent tirer, d'autant que leurs camarades les conseillent pour se placer sur le terrain, les mettent en position favorable pour agir, etc. (Grissault, 2006).

\subsection{La durée de l'activité}

Déterminer la durée de l'activité nécessaire à un élève pour développer une habileté motrice ou développer une ressource physique implique de prendre en compte certaines caractéristiques fréquemment avancées concernant des jeunes avec un retard mental :

- la lenteur d'exécution, en accordant un temps d'activité conséquent, tout en veillant à accroître progressivement, autant que possible, la contrainte temporelle ;

- le besoin de répéter une même situation d'apprentissage, pour stabiliser les acquis ;

- de faibles ressources bioénergétiques, qui peuvent aller de pair avec un surpoids ou une obésité (Eberhard, 2009), et qui appellent des temps de sollicitation de ces ressources qui soient adaptés, ainsi que des temps de récupération suffisants ;

- la difficulté à maintenir la concentration sur une tâche.

\subsection{Des responsabilités à assumer}

Être à l'origine d'un choix ou d'une action participe de la responsabilité personnelle, contribue à une meilleure estime de soi et à une autonomie accrue. Cet effet positif peut être renforcé si ce qui est imputable au sujet relève d'un rôle socialement reconnu, dont il a à répondre devant les autres et qui lui vaut un regard positif s'il parvient à l'assumer. Si l'on admet que "la condition essentielle pour que ces personnes — avec une déficience intellectuelle, ndlr puissent accéder à une identité socialement valorisée est de prouver qu'elles peuvent être utiles » (Diederich, 2004, 35), une contribution au fonctionnement du groupe s'avère alors particulièrement pertinente. 
Confier des responsabilités suppose de faire confiance. Lorsqu'on s'adresse à des jeunes ayant des troubles importants des fonctions cognitives, il est légitime de se demander s'ils sont prêts à exercer celles que l'on envisage, mais il faut poser que la responsabilité s'apprend. Son niveau de difficulté, ainsi qu'un éventuel accompagnement par un tiers, sont à décider en fonction de chaque élève, pour des rôles concernant par exemple la gestion d'une activité : responsable du matériel — mettre en place et ranger... - ; observateur — au basket-ball, compter le nombre de tirs réussis, cocher le nom des joueurs qui marquent des points... ; arbitre — plutôt en coarbitrage ; pareur — en gymnastique ; responsable de l'échauffement précédant une activité, etc.

\subsection{La structuration de la séance}

Une réponse au besoin de stabilité de certains élèves est à chercher dans une même structuration des séances composant un cycle d'activité. Parmi les repères fixes constituant un rituel rassurant, on a par exemple le rappel de ce qui a été fait lors de la séance précédente, la présentation de la séance du jour, un échauffement conduit de façon identique, une même disposition du matériel, son rangement par les mêmes élèves...

\section{L'ACCOMPAGNEMENT DE L'ELEVE DURANT LA SEANCE D'EPS}

Il s'agit là d'envisager le rôle, durant la séance, de l'enseignant et éventuellement des personnes qui sont présentes - AVS, enseignant de l'Ulis, autres élèves.

\subsection{Des procédures de guidage complémentaires}

Un mode d'enseignement basé sur la reproduction de modèles gestuels a souvent fait place à une pédagogie valorisant l'adaptation du sujet et l'aménagement matériel de l'environnement. Cette orientation ne doit pas conduire à méconnaître le rôle des interactions humaines dans l'apprentissage, notamment pour les habiletés motrices. Les travaux de Winnykamen et Lafont, prolongeant ceux de Bandura, ont montré que l'observation d'autrui mobilisait des processus attentionnels, mnésiques, motivationnels et de reproduction motrice. Mais la pertinence des modalités de guidage dépend du type d'habileté à acquérir.

Quand il s'agit d'habiletés dites fermées, en jeu lors de tâches effectuées dans des conditions environnementales stables, sans incertitude — danse, gymnastique...- - et dont le but est de reproduire des formes gestuelles, le pratiquant doit fixer un schème d'action conforme au modèle. À cet effet, la démonstration explicitée du geste s'avère supérieure à l'aménagement matériel.

En revanche, dans le cas d'habiletés ouvertes, correspondant à des tâches réalisées dans un environnement incertain - l'incertitude pouvant tenir à l'environnement physique, dans des activités de pleine nature, ou humain, dans les sports d'opposition - la diversification des schèmes d'action et un guidage par la connaissance des résultats se révèlent efficaces. En fait, 
ce type d'habileté peut aussi faire appel à des gestes techniques. Pour traiter le problème posé par une tâche, plusieurs de ces gestes sont susceptibles d'être retenus. L'apprentissage peut alors bénéficier d'une démonstration de techniques pouvant convenir à l'élève, ainsi que de consignes d'actions.

Au final, les procédures de guidage apparaissent complémentaires, selon la nature de la tâche, le contexte et les caractéristiques du sujet (Lafont, 2006). Ces données, obtenues à partir d'études portant sur des individus ordinaires, sont exploitables avec des personnes présentant une déficience intellectuelle, en sachant que la pertinence des procédures retenues ne peut être établie qu'au cas par cas, à partir de l'observation de l'élève engagé dans une activité.

\subsection{La communication}

Dans la mesure où certaines consignes peuvent être présentées par une démonstration de la tâche à effectuer, la compréhension est a priori moins difficile en EPS qu'ailleurs. Mais faute de comprendre la consigne orale, l'élève risque d'imiter ses camarades et de ne pas s'engager dans la recherche de ses propres solutions au problème posé — par exemple pour une activité d'opposition duelle ou collective. Par ailleurs, dans une perspective éducative qui ne se limite pas à la motricité, il est important de renforcer la compréhension et l'usage de la langue chez les élèves avec un handicap mental. La sollicitation du canal visuel doit donc s'accompagner d'une mise en mots de ce qui est montré et vécu lors de l'activité.

Pour se faire comprendre, le nécessaire recours à un lexique et à une syntaxe suffisamment simples n'est pas à confondre avec l'adoption d'un langage infantile. L'enseignant peut procéder à des reformulations, accompagner son message oral de gestes appropriés et redoubler une consigne délivrée à l'ensemble du groupe par une consigne adaptée, adressée à un élève en particulier. En outre, pour certaines tâches, des dessins représentant ce qui est à faire peuvent être disposés sur le terrain.

La communication ne se limite pas aux consignes. Solliciter la réflexion des élèves sur une activité exige d'adapter le niveau d'abstraction des messages et la complexité des situations à analyser. Faire porter l'attention sur des éléments peu nombreux et objectivés contribue à diminuer les difficultés d'abstraction. Si, par exemple, on veut inviter à comparer la qualité de réalisation d'une même figure gymnique réalisée par deux élèves, des questions précises peuvent y conduire progressivement en retenant des critères peu équivoques.

Communiquer n'est pas seulement le fait de l'enseignant. Toutes les occasions susceptibles de permettre à l'élève de s'exprimer sont à rechercher, et la prise de risque inhérente à cette manifestation personnelle mérite d'être valorisée, quelle que soit la pertinence de ce que qui est exprimé, car elle témoigne d'une confiance en soi favorable à l'autonomie.

\subsection{Des médiations socio-affectives}

Des médiations socio-affectives s'imposent quand l'élève doit être mis en confiance ou encouragé pour oser s'engager dans une activité, persévérer dans son effort, s'exprimer afin de formuler un avis, un choix, ou lorsque ses réussites demandent à être valorisées, ses prises de 
risque canalisées, ses relations avec les autres régulées, et son attitude harmonisée avec les obligations de la vie collective.

Dans le cas d'un retard mental, l'expérience d'un individu face à des apprentissages a souvent été marquée par l'échec. Face à de nouvelles situations d'apprentissage, s'attendant à un nouvel échec et le craignant, on peut redouter qu'il ne se mobilise pas pour réussir. La démobilisation est renforcée par une tendance à considérer que la réalité échappe à son contrôle, à attribuer sa réussite davantage à des causes extérieures — des encouragements, l'enseignant, la chance qu'à ce qui relève de son action propre. Dans ces conditions, dès lors que ses efforts ne débouchent pas sur des résultats immédiatement valorisants, l'élève en vient à renoncer à ses efforts (Magerotte, 1984, 18). Se découvrir capable d'agir avec succès et de progresser est donc fondamental pour des jeunes qui ont des difficultés à se reconnaître un pouvoir d'emprise sur les événements, de même que relativiser les difficultés rencontrées, pour que la frustration n'entraîne pas un sentiment d'échec persistant.

La valorisation des réussites passe notamment par leur externalisation (Bruner, 1996, 39), leur exposition devant des tiers. Les progrès, les $2 u v r e s$ produites, pourront ainsi devenir, aux yeux de l'enfant ou de l'adolescent et aux yeux des autres, des sources de reconnaissance de sa capacité d'agir (Garel, 2006). Produire une œuvre par son activité physique, c'est donner à voir ses résultats, et si possible en laisser une trace. Les activités à caractère artistique en donnent l'occasion, mais elles ne sont pas les seules. Toute activité peut être montrée aux autres selon des modalités diverses — dans l'instant, en différé par une photo... Et l'évaluation peut être consignée sur une fiche ou un cahier.

Concernant les relations entre élèves, il convient de veiller à ce que la présence d'élèves handicapés ne soit pas une source de tensions, et un tutorat ne doit pas se traduire chez l'élève tuteur par une attitude surprotectrice ou par une saturation provoquée par cette responsabilité, ou encore par le refuge dans ce rôle pour échapper aux contraintes de sa propre activité.

\subsection{La sollicitation de processus cognitifs}

Une altération des capacités intellectuelles ne signifie pas leur extinction chez tous les individus. Il est donc utile de les solliciter, en faisant le pari que des médiations visant les processus cognitifs entraîneront leur mobilisation et en accroîtront les performances.

\subsubsection{L'orientation et le maintien de l'attention}

Les capacités perceptives et attentionnelles étant diminuées, l'enseignant, ou plus généralement un tiers, peut attirer et maintenir l'attention de l'élève sur ce qui est à faire et sur un élément de la situation à prendre en compte pour réussir une tâche. Il s'agit donc que le jeune soit confronté à des objectifs précis, présentés pour le court terme, et que l'environnement soit structuré de façon à pouvoir y percevoir des aspects importants.

Les difficultés d'attention appellent le recours à des signaux de régulation de l'activité — ou feedback - (Magerotte, op. cit.) relativement fréquents et immédiats : le sujet produit telle 
action et, à partir de l'environnement physique ou humain, il est informé du résultat de son entreprise.

\subsubsection{Au-delà de la stabilisation des acquis, la généralisation des apprentissages}

Chez des enfants et adolescents avec un retard mental, stabiliser les acquis d'un apprentissage peut demander de se remettre à l'ouvrage plus qu'avec une population ordinaire. Toutefois, s'en tenir à ce constat risque de méconnaître un objectif qui peut sembler antinomique alors qu'il est complémentaire : généraliser les apprentissages, en d'autres termes en favoriser le transfert pour construire des compétences. L'acquisition de compétences suppose en effet de pouvoir transférer ce qui a été appris dans une situation à une autre qui relève d'un même type de problème - le démarquage en sports collectifs, par exemple.

S'efforcer de libérer l'activité de l'élève de son contexte initial, viser l'adaptabilité, est paradoxal pour des jeunes dont le retard mental appelle un contexte d'activité stable. Cet objectif est important, car leurs capacités d'adaptation sont déficitaires : "leurs modalités perceptives et exploratoires sont peu propices à la prise en compte de la nouveauté. D'où l'absolue nécessité de réaliser les apprentissages dans diverses conditions de stimulation, dont les différences pourront être progressivement accentuées, mais dont on fera apparaître les éléments communs » (Magerotte, op. cit., 21). Il ne faut pas se cacher que cet objectif est parfois bien difficile à atteindre dans le cas des déficiences harmoniques, qui se distinguent des déficiences dysharmoniques notamment par de très faibles capacités d'adaptation, "orientées selon des stéréotypes rigides et peu mobilisables » (Misès, Perron \& Salbreux, 1997, 1555).

\subsubsection{L'évaluation portée par l'élève sur son activité}

Dans la mesure du possible, l'élève doit être amené à identifier le résultat de son action grâce à des indicateurs de réussite peu nombreux, appréhendés à partir de repères suffisamment simples et concrets, par exemple des zones de réception matérialisées lors d'un saut en longueur, un foulard à saisir ou une clochette à actionner en escalade, le nombre d'actions réussies sur le nombre d'actions tentées... Cette évaluation permet à l'élève de se situer :

- par rapport à une performance présente — «voilà ce que j'arrive à faire »-, ce qui contribue à sa conscience de la réalité, à distance d'un sentiment d'incapacité sclérosant ou, au contraire, d'un sentiment de toute-puissance illusoire ;

- par rapport à une performance passée, ce qui peut lui fait prendre conscience de ses éventuels progrès ;

- par rapport à des niveaux de réalisation possibles, ce qui suppose de hiérarchiser par ordre de difficulté les indicateurs de réussite retenus, par exemple, sur le thème de l'équilibre, en prenant en compte la hauteur et la largeur de la surface de déplacement, la direction du déplacement, sa continuité, sa vitesse...

- par rapport à une performance à venir, ce qui donne la possibilité de s'engager dans un projet d'apprentissage à partir d'une connaissance de niveaux de performance pas encore atteints mais envisageables ; 
- par rapport à une modalité de réalisation — « en m'y prenant de telle ou telle manière...-, ce qui peut conduire à une mise en relation d'un résultat obtenu avec la procédure utilisée pour l'obtenir, et donc à une réflexion méthodologique.

\subsubsection{Une réflexion méthodologique}

Conduire l'élève à établir un lien entre son action et ses conséquences, à dégager entre ces deux éléments une relation de cause à effet permettant d'apprécier la pertinence de ce qui a été fait en fonction du but recherché, relève d'un objectif de méthode conduisant l'élève à connaître les modalités et les contextes d'action qui lui sont le plus favorables. Il peut s'agir, par exemple, de comparer deux façons de lancer une balle et de déterminer celle qui permet de toucher le plus souvent une cible.

Cette démarche permet aussi au jeune de relativiser un échec, pas en se leurrant sur ce qu'il a réalisé, mais dans le sens où le résultat de son action peut être mis en relation avec la manière dont il a agi. Il est important que l'échec ne soit pas considéré comme une fatalité liée à sa personne et à son irréductible impuissance, mais à des façons de faire sur lesquelles il a prise.

Une telle approche méthodologique participe du développement des capacités stratégiques du sujet, dont on sait qu'elles sont inférieures à la normale dans le cas d'une déficience intellectuelle. Certes, le retard mental est parfois si important que la démarche préconisée bute sur des limites infranchissables, mais croire a priori que les ressources cognitives d'un individu ne peuvent pas être sollicitées en raison de ses difficultés participerait d'une représentation propre à l'enfermer dans ses manques. Pour qui est en grande difficulté intellectuelle, il se peut que l'on doive s'en tenir à solliciter le oui ou le non, l'adhésion ou le refus, ce qui constitue déjà pour certains un élément d'autonomie appréciable.

\subsection{L'autonomisation}

L'autonomie, ou plutôt l'autonomisation, car il s'agit d'un processus jamais tout à fait achevé, est un objectif fréquemment mentionné dans les projets éducatifs, d'autant plus que les personnes avec une déficience intellectuelle sont loin d'être en toute circonstance capables d'agir sans aide, et même de s'autoriser une part d'autonomie. Devant sa culpabilité d'être né ainsi, il arrive qu'un adolescent handicapé mental se conforme à " une image qui définit la manière dont il doit être, dont il doit se comporter» (Michel, 2009, 177). Pour certaines personnes, s'engager à agir de soi-même est problématique, car elles sont « dominées par une inhibition particulière et déstabilisante (...). Elles ne cherchent pas à prendre du plaisir (...). Toute non-directivité génère une angoisse catastrophique » (ibid., 155).

\subsubsection{L'invitation au choix}

Les situations permettant à l'élève d'exprimer un choix sont recherchées, qu'il s'agisse de choisir un parcours à sa mesure parmi d'autres de difficulté variable, de décider d'une stratégie, de donner son point de vue sur la qualité d'une production, lors d'une activité artistique, en l'argumentant dans la mesure du possible, etc. 


\subsubsection{Une juste distance}

Pour le jeune qui ne s'engage ou ne se maintient dans une activité qu'en lien avec un partenaire ou un adulte donné, il s'agit d'apprendre progressivement à agir à distance de cette présence rassurante. L'autonomisation nécessite en effet une juste distance par rapport à la personne qui l'accompagne, ni trop près ni trop loin d'elle. L'enseignant peut être trop aidant, envahissant, imposant une présence qui entrave la capacité du sujet à agir par lui-même (Garel, 2007). Une juste distance est d'autant plus délicate à fixer qu'elle est variable selon les individus et les situations.

\subsubsection{Une notion relative}

Si l'on pose qu'un individu est autonome dans la mesure où il est capable " de se diriger tout seul sans être contraint par quelqu'un d'autre à se conduire comme il le fait » (Descombes, 2004, 442), alors il fait bien preuve d'autonomie dès lors que la personne aidante ne se substitue pas à lui pour imposer son aide et, plus généralement, prendre des décisions à sa place. Dans ces conditions, on peut considérer que "l'autonomie consiste peut-être moins à être indépendant qu'à choisir et gérer ses dépendances» (Sticker, 1997, 21), qu'elle peut s'exprimer par des demandes d'aide, de conseil, d'accompagnement. Ce qui suppose que la personne soit accompagnée pour prendre conscience de ce qu'elle sait et peut faire, puis qu'elle ose et puisse faire appel à autrui en cas de besoin.

\section{POUR CONCLURE : UNE ATTENTION MESUREE AUX DIFFERENCES}

Les préconisations avancées ne doivent pas occulter qu'elles concernent une population très hétérogène : entre les différents syndromes, la diversité des atteintes cognitives comme des capacités, et la singularité des histoires individuelles, il y a de quoi les accueillir avec prudence. Mais elles se veulent suffisamment ouvertes pour ne pas être confondues avec un prêt-à-porter, organisationnel et pédagogique, qui s'appliquerait parfaitement à tout individu et à tout type de situation d'enseignement. La complexité des situations effectivement rencontrées est telle qu'il convient de réajuster les modèles à une réalité toujours particulière.

S'attacher à la réalité, notamment aux besoins spécifiques d'un élève, implique d'être attentif à son activité face aux tâches proposées. Cette observation doit conduire à identifier ses difficultés et ses capacités, les obstacles qu'il rencontre (Garel, 2009), les procédures qu'il utilise pour les franchir, les modalités d'accompagnement de son activité qui lui conviennent et ce qui peut le mobiliser.

Cette approche personnalisée, empreinte d'un souci d'équité pour corriger par des mesures appropriées ce qu'un enseignement fondé sur un principe d'égalité formelle peut avoir d'injuste, ne doit toutefois pas conduire à une pédagogie des différences (Bui-Xuân \& Mikulovic, 2007). Si l'attention aux différences est nécessaire, elle doit s'accompagner d'une attention aux ressemblances, car si une personne en situation de handicap a des singularités, comme tout un chacun, à des degrés divers, elle peut partager plus ou moins avec les valides 
des caractéristiques semblables, en termes de besoins, de désirs, etc., qui invitent à ne procéder à des adaptations de l'enseignement qu'à hauteur de ce qu'il est nécessaire, sans en rabattre sur les ambitions que l'on peut nourrir à l'égard de cette personne (Garel, 1999).

Certes, il faut rester lucide sur les capacités des personnes en situation de handicap, mais le fait est qu'on les sous-estime trop souvent. Il est important que l'école joue en leur faveur pleinement son rôle, qui tient à ce qu'elle est un lieu privilégié pour satisfaire à des enjeux d'accomplissement personnel et de participation sociale, car elle s'adresse, normalement, à tous les enfants et adolescents, à un âge donc où se construit l'avenir.

\section{BIBLIOGRAPHIE}

Baron M., Brunet F. \& Verret C. (2005). Gérer au quotidien son autonomie fonctionnelle. Activités physiques adaptées aux personnes en perte d'autonomie, Dossier EP.S n ${ }^{\circ} 63$, Éd. Revue EP.S.

Bruner J. (1996). L'éducation, entrée dans la culture. Les problèmes de l'école à la lumière de la psychologie culturelle, Paris, Retz.

Brunet F., Bui-xuân G. \& Bluteau S. (2001). Enseigner et animer les activités physiques adaptées aux personnes déficientes intellectuelles, in A. Varray, J. Bilard \& G. Ninot, Enseigner et animer les activités physiques adaptées, Dossier EP.S n55, Édit. Revue EP.S, 134-142.

Brunet F. \& Mautuit D. (2003). Activités physiques adaptées aux personnes déficientes intellectuelles. Du programme institutionnel au projet personnalisé, dossier EP.S n ${ }^{\circ}$ 60, Éd. Revue EP.S.

Bui-xuân G. \& Mikulovic J. (2007). Les élèves à besoins particuliers n'ont pas besoin d'une pédagogie particulière, Reliance, $\mathrm{n}^{\circ} 24,98-106$.

Corps, mouvement, déficience mentale, société. (1984). Actes des journées d'études du CTNERHI, Strasbourg, 8/9/10 nov., Paris, Édit. du CTNERHI.

Descombes V. (2004). Le complément de sujet. Enquête sur le fait d'agir de soi-même, Paris, Gallimard.

Diederich N. (2004). Les naufragés de l'intelligence, $2^{\mathrm{ème}}$ édition, Paris, La découverte.

Eberhard Y. (2009). Intégrer/inclure en cours d'EPS les enfants avec des déficiences intellectuelles, actes du colloque DGESCO/ESEN, Mai 2009. Disponible sur le site : http://www.esen.education.fr/fr/ressources-par-type/conferences-en-

ligne/regroupements-de-conferences/scolarisation-des-eleves-handicapes-et-educationphysique-et-sportive/

Garel J.-P. (1999). Individualiser pour réunir : l'enseignement de l'EPS devant un paradoxe de l'intégration scolaire, in J. Gateaux-Mennecier \& M.-C. Mège-Courteix (dir.), Marginalisation, intégration, La nouvelle revue de l'adaptation et de la scolarisation, $\mathrm{n}^{\circ}$ 8, INS HEA, 153-165.

Garel J.-P. (2001). Des collégiens parmi les autres. L'intégration en éducation physique et sportive d'élèves présentant un handicap mental, Document audiovisuel, INS HEA, $40^{\prime}$. 
Garel J.-P. (2006). La reconnaissance de la capacité d'agir sur et par un corps altéré, enjeu de la construction du sujet, in A. Marcellini (dir.), Handicaper : éducation corporelle et handicap, Éduquer, $\mathrm{n}^{\circ} 11$, Paris, L’harmattan, 61-71.

Garel J.-P. (2007). L'autonomie de collégiens présentant un retard mental : ses conditions de possibilité en éducation physique et sportive, Reliance, $\mathrm{n}^{\circ} 24,36-48$.

Garel J.-P. (2009). EPS et situations de handicap : projets personnalisés et activités communes, in F. Brunet, C. Blanc \& A.-C. Margot (dir.), Polyhandicap-Handicap sévère - Activités motrices et sensorielles, Éd. Actio, Joinville le Pont, 131-146.

Grissault C. (2006). Exemple d'intégration d'élèves d'UPI en EPS. Disponible sur : http://eps.ac-orleans-

tours.fr/php5/eps_adaptee/integration_d_eleves_d_upi_en_eps.htm

Joly K. (2006). Mises en cuvre pédagogiques pour favoriser le lien social lors d'une intégration d'élèves de l'U.P.I. en E.P.S., mémoire 2 CA-SH, option D.

Lafont L. (2006). Entre démonstration et auto-régulation. Le rôle de procédures de guidage ajustées aux caractéristiques des apprenants : interaction de tutelle, imitationmodélisation interactive et coping modèles, 7e colloque européen sur l'autoformation, Faciliter les apprentissages autonomes, Toulouse, ENFA, 18-20. Disponible sur : http://www.enfa.fr/autoformation/

Magerotte G. (1984). La personne déficiente mentale : apprentissage et activités physiques et sportives, in Corps, mouvement, déficience mentale, société, Actes des journées d'études du CTNERHI, Strasbourg, Édit. du CTNERHI, 8/9/10 nov., 13-24.

Meirieu P. (1987). Apprendre, oui mais comment? Paris, ESF.

Michel F., Handicap mental : crime ou châtiment? Paris, Coéd. Le Monde- Puf, 2009.

Mises R., Perron R. \& Salbreux R. (1994). Retards et troubles de l'intelligence de l'enfant, Paris, ESF. 